 \\ MOUNTaiNS \\ Mathematical Publications
}

\section{PERIOD ANNULI AND MULTIPLE SOLUTIONS FOR TWO-POINT BVP}

\author{
Svetlana Atslega - Felix Sadyrbaev
}

\begin{abstract}
In this article we consider equations of the type $x^{\prime \prime}+g(x)=0$ and $x^{\prime \prime}+f(x) x^{\prime 2}+g(x)=0$. The Neumann boundary value problem is considered. For polynomials $f$ and $g$ we provide the multiplicity results. These results are based on a thorough analysis of a phase plane. The existence of period annuli is concerned.
\end{abstract}

\section{Introduction}

We consider first autonomous equation

$$
x^{\prime \prime}+g(x)=0
$$

with respect to solutions which satisfy the Neumann boundary conditions

$$
x^{\prime}(0)=0, \quad x^{\prime}(1)=0 .
$$

Our intent is to study families of periodic solutions and formulate conditions which ensure the existence of solutions to the Neumann problem. Equation (1) is equivalent to the system

$$
x^{\prime}=y, \quad y^{\prime}=-g(x) .
$$

Integrating equation (11) gives the relation

$$
x^{\prime 2}+2 G(x)=C,
$$

where $C$ is a constant, $G(x)=\int_{0}^{x} g(s) \mathrm{d} s$. Let $x(t)$ be a solution of (11). A constant $C$ may be identified with $x^{\prime 2}(0)$ if $x(0)=0$ or with $2 G\left(x_{e k s t r}\right)$ where $x_{e k s t r}$ is an extremal value of $x$. Suppose that $x(t)$ is a solution which satisfies the initial conditions

$$
x(0)=x_{0}, \quad x^{\prime}(0)=0 .
$$

2000 Mathematics Subject Classification: 34B15, 34C25, 34C37.

Keywords: Neumann boundary conditions, phase portrait, period annulus, multiplicity of solutions. 
Then $x^{\prime 2}(t)+2 G(x(t))=2 G\left(x_{0}\right)$ for any $t$. Therefore $\frac{\mathrm{d} x}{\mathrm{~d} t}=\sqrt{2 G\left(x_{0}\right)-2 G(x(t))}$ for increasing solution. If the function $G\left(x_{0}\right)-G(x)$ is positive for $x \in\left(x_{0}, x_{1}\right)$ and $G\left(x_{0}\right)=G\left(x_{1}\right)$ then:

- equation (11) has a periodic solution with a phase orbit passing through the points $\left(x_{0}, 0\right)$ and $\left(x_{1}, 0\right)$;

- equation (11) has a solution which satisfies the conditions

$$
x(0)=x_{0}, \quad x(T)=x_{1}, \quad x^{\prime}(t)>0 \quad \text { for } \quad t \in(0, T) ;
$$

- equation (11) has a solution which satisfies the conditions

$$
x^{\prime}(0)=0, \quad x^{\prime}(T)=0 .
$$

A constant $T$, which will be called half-period, can be calculated and the value is

$$
T\left(x_{0}\right)=\frac{1}{\sqrt{2}} \int_{x_{0}}^{x_{1}} \frac{\mathrm{d} s}{\sqrt{G\left(x_{0}\right)-G(s)}} .
$$

Let us consider two kinds of families of periodic solutions. Suppose that $p$ is a zero of $g(x)$ such that $g^{\prime}(p)>0$. One easily can verify that the point $(p, 0)$ is a critical point of the system (3) and this point is a center. This point has a punctured neighborhood covered with nontrivial cycles. Due to terminology in $\mathrm{S}$ a b a t i n i [3], the largest connected region covered with cycles surrounding $(p, 0)$ is called a central region. Every connected region covered with nontrivial concentric cycles is called a period annulus. We will call a period annulus associated with a central region by a trivial period annulus. Periodic trajectories of a trivial period annulus encircle exactly one critical point of the type center. Period annuli enclosing several (more than one) critical points will be called nontrivial period annuli, respectively.

Three central regions (trivial period annuli) and nontrivial period annulus are depicted in Figure 2.

Conditions for existence of a nontrivial period annulus can be given in terms of the primitive (anti-derivative) function $G(x)$. If $G(x)$ has two non-neighboring points of maximum then a period annulus exists and its location may be described precisely.

If behavior of the function $T\left(x_{0}\right)$ defined on a period annulus is known then conclusions about the existence of solutions to the Neumann problem (11), (2) can be made. If minimum of $T(x)$ over a period annulus is much less than the length of the interval $[0,1]$ then multiple solutions of the Neumann BVP are expected associated with this period annulus.

This is briefly what concernes the Neumann BVP for equation (1).

The next object of investigation is the Liénard type equation

$$
x^{\prime \prime}+f(x) x^{\prime 2}+g(x)=0 .
$$


The theory of equations of the form $x^{\prime \prime}+f(x) x^{\prime}+g(x)=0$ is of great interest both from theoretical and practical point of view. Especially investigation of isolated periodic solutions (limit cycles) is challenging even if $g$ and $f$ are polynomial functions. We consider equation in the form (17) with $x^{\prime 2}$ in the middle term. Then:

- phase portraits are symmetric with respect to the axis $x$;

- isolated periodic solutions do not exist;

- multiple period annuli may exist instead.

Important fact about equations in the form (7) is that they can be reduced to the form $u^{\prime \prime}+h(u)=0$ by the simple change of dependent variable

$$
u=\int_{0}^{x} e^{F(s)} \mathrm{d} s, \quad F(x)=\int_{0}^{x} f(s) \mathrm{d} s .
$$

A number of papers deal with equation

$$
x^{\prime \prime}+f(x) x^{\prime}+g(x)=0,
$$

considering oscillatory behavior of solutions and looking for isolated periodic solutions - limit cycles. We look for non-isolated periodic solutions. Our goal in this paper is to give efficient criteria for the existence of multiple period annuli.

Definitions. Consider the equivalent two-dimensional differential system

$$
x^{\prime}=y, \quad y^{\prime}=-f(x) y^{2}-g(x) .
$$

It has critical points at $\left(p_{i}, 0\right)$, where $p_{i}$ are zeros of $g(x)$. Recall that a critical point $\mathrm{O}$ of (3) is a center if it has a punctured neighborhood covered with nontrivial cycles. Due to terminology in $\mathrm{S}$ a b a t i $\mathrm{n}$ i [3], the largest connected region covered with cycles surrounding $\mathrm{O}$ is called a central region. Every connected region covered with nontrivial concentric cycles is usually called a period annulus. We will call a period annulus associated with a central region by a trivial period annulus. Periodic trajectories of a trivial period annulus encircle exactly one critical point of the type center. Period annuli enclosing several (more than one) critical points will be called nontrivial period annuli, respectively.

We are looking for multiple nontrivial period annuli in equation.

First we consider equation (7) with $f \equiv 0$. We show that period annuli exist if the primitive (anti-derivative) $G(x)=\int^{x} g(s) \mathrm{d} s$ has non-neighbouring points $M_{1}$ and $M_{2}$ of local maxima such that values of $G$ at $M_{1}$ and $M_{2}$ are strictly greater than those at intermediate points of maxima.

In order to pass to equation (7) with non-zero functions $f$ we use transformation by S a batini [4] which allows the reduction of equation (7) to a conservative one of the form

$$
u^{\prime \prime}+h(u)=0,
$$


where periodic solutions $x(t)$ of (7) are in one-to-one correspondence with periodic solutions $u(t)$. The behaviour of the function $g(\xi) e^{2 F(\xi)}$ is crucial for existence of period annuli in both equations (7) and (11).

\section{Equation $x^{\prime \prime}+g(x)=0$}

We assume that $g(x)$ is a polynomial with simple zeros, that is, if $g(z)=0$ then $g^{\prime}(z) \neq 0$.

The primitive $G(x)=\int_{0}^{x} g(s) \mathrm{d} s$ may have multiple maxima. It is easy to observe that the equivalent differential system

$$
x^{\prime}=y, \quad y^{\prime}=-g(x)
$$

has centers at the points $\left(m_{i}, 0\right)$ and saddle points at $\left(M_{j}, 0\right)$, where $m_{i}$ and $M_{j}$ are points of local minima and maxima, respectively.

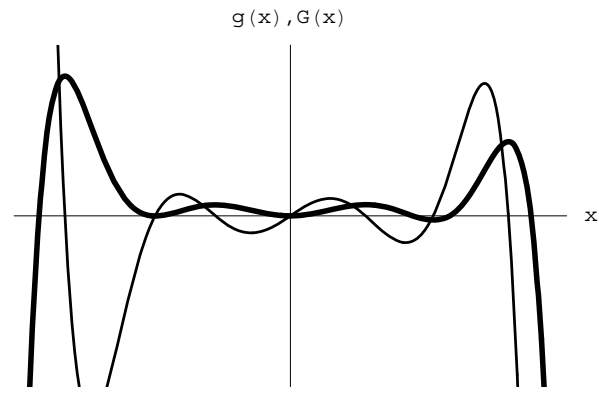

FIGURE 1.

Functions $g(x)$ and the primitive $G(x)$ (bold).

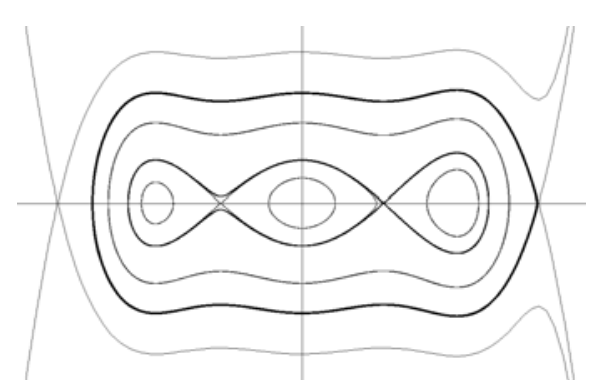

Figure 2.

The phase plane.

Proposition 2.1. Critical points of the system (12) are "saddles" and "centers" which alternate.

TheOREM 2.1. Let $M_{1}$ and $M_{2}\left(M_{1}<M_{2}\right)$ be non-neighbouring points of maximum of the function $G(x)$. Suppose that interior (with respect to the interval $\left.\left(M_{1} ; M_{2}\right)\right)$ maxima of $G$ are strictly less than $\min \left\{G\left(M_{1}\right) ; G\left(M_{2}\right)\right\}$.

Then there exists at least one nontrivial period annulus.

Pr o of. Consider the case $G\left(M_{1}\right)>G\left(M_{2}\right)$.

There are at least two critical points of the type "center" in the interval $\left(M_{1} ; M_{2}\right)$. We will show that there exists a homoclinic solution emanating from the point $\left(M_{2} ; 0\right)$ and enclosing all critical points in the interval $\left(M_{1} ; M_{2}\right)$. 
Consider the primitive $G_{M_{2}}(x)=\int_{M_{2}}^{x} g(s) \mathrm{d} s$. Let $r$ be the first zero of $G_{M_{2}}(x)$ to the left of $M_{2}$. Consider the trajectory defined by the relation

$$
x^{\prime 2}(t)+2 G_{M_{2}}(x(t))=2 G_{M_{2}}\left(M_{2}\right)
$$

which passes through the point $(r ; 0)$. Let $T$ be the time needed for the point $(r ; 0)$ to move to a position $\left(M_{2} ; 0\right)$ along the trajectory. This time is given by the formula

$$
T=\int_{r}^{M_{2}} \frac{\mathrm{d} s}{\sqrt{2 G_{M_{2}}\left(M_{2}\right)-2 G_{M_{2}}(s)}}
$$

Notice that $G^{\prime}(s)=g(s), g\left(M_{2}\right)=0, g^{\prime}\left(M_{2}\right)<0$. One obtains then the relation

$$
\begin{aligned}
G_{M_{2}}\left(M_{2}\right)-G_{M_{2}}(s) & =-\frac{1}{2} G_{2}^{\prime \prime}\left(M_{2}\right)\left(s-M_{2}\right)^{2}+o\left(\left(s-M_{2}\right)^{2}\right) \\
& =-\frac{1}{2} g^{\prime}\left(M_{2}\right)\left(s-M_{2}\right)^{2}+o\left(\left(s-M_{2}\right)^{2}\right),
\end{aligned}
$$

where $o(\xi) \rightarrow 0$ as $\xi \rightarrow 0$. Then

$$
\begin{aligned}
T & =\int_{r}^{M_{2}} \frac{\mathrm{d} s}{\sqrt{-g^{\prime}\left(M_{2}\right)\left(s-M_{2}\right)^{2}+o\left(\left(s-M_{2}\right)^{2}\right)}} \\
& =\frac{1}{\sqrt{-g^{\prime}\left(M_{2}\right)}} \int_{r}^{M_{2}} \frac{\mathrm{d}\left(s-M_{2}\right)}{\sqrt{\left(s-M_{2}\right)^{2}+o\left(\left(s-M_{2}\right)^{2}\right)}} \\
& =\frac{1}{\sqrt{-g^{\prime}\left(M_{2}\right)}} \int_{r-M_{2}}^{0} \frac{\mathrm{d} \xi}{\sqrt{\xi^{2}+\xi^{2} o(\xi)}} \\
& =\frac{1}{\sqrt{-g^{\prime}\left(M_{2}\right)}} \int_{r-M_{2}}^{0} \frac{\mathrm{d} \xi}{|\xi| \sqrt{1+o(\xi)}} \\
& >\frac{1}{\sqrt{-g^{\prime}\left(M_{2}\right)}} \int_{-\delta}^{0} \frac{\mathrm{d} \xi}{|\xi| \sqrt{1+\frac{1}{2}}} \\
& =\frac{\sqrt{2}}{\sqrt{-3 g^{\prime}\left(M_{2}\right)}} \int_{-\delta}^{0} \frac{\mathrm{d} \xi}{|\xi|}=+\infty
\end{aligned}
$$

where $\delta>0$ is such that $|o(\xi)|<\frac{1}{2}$ for $\xi \in[-\delta, 0]$. Any trajectory of equation (13) is symmetric with respect to the $x$-axis. Therefore a homoclinic solution associated with the point $\left(M_{2}, 0\right)$. 
Let $M_{*}$ be a point of the greatest interior maximum of $G(x)$ in the interval $\left(M_{1}, M_{2}\right)$. By our assumptions $G\left(M_{1}\right)>G\left(M_{2}\right)>G\left(M_{*}\right)$. Let $r_{*}>M_{1}$ be a nearest to $M_{1}$ point such that $G\left(r_{*}\right)=G\left(M_{*}\right)$. Any trajectory starting from a point $(s, 0)$, where $s \in\left(r, r_{*}\right)$, is described by the relation

$$
x^{\prime 2}(t)+2 G(x(t))=2 G(s)
$$

and therefore intersects the $x$-axis at some point $s_{1} \in\left(M_{* *}, M_{2}\right)$, where $M_{* *}<M_{2}$ is a nearest to $M_{1}$ point such that $G\left(M_{* *}\right)=G\left(M_{*}\right)$. A collection of these trajectories forms a period annulus which encloses all the critical points in the interval $\left(M_{1}, M_{2}\right)$.

The case of $G\left(M_{1}\right)<G\left(M_{2}\right)$ can be considered similarly. In the case of $G\left(M_{1}\right)=G\left(M_{2}\right)$ we have $r=M_{1}$ and a heteroclinic trajectory appears which connects the points $\left(M_{1}, 0\right)$ and $\left(M_{2}, 0\right)$. In other respects the proof is the same.

\section{The Neumann problem}

Consider the problem

$$
\begin{gathered}
x^{\prime \prime}+g(x)=0, \\
x^{\prime}(a)=0, \quad x^{\prime}(b)=0 .
\end{gathered}
$$

Theorem 3.1. Suppose equation (18) has a period annulus $C$. Let $C_{x}$ be intersection of a period annulus $C$ with the $x$-axis (this intersection is a sum of two open intervals $I_{1}$ and $\left.I_{2}\right)$. Let $T\left(x_{0}\right)$ stand for a half-period of a periodic solution which belongs to $C, x_{0} \in\left(x_{0}^{*}, x_{0}^{* *}\right)=: I_{1}$. Let $k$ be the largest integer such that

$$
k T_{\min }<b-a<(k+1) T_{\min }, \quad T_{\min }=\min \left\{T\left(x_{0}\right): x_{0} \in\left(x_{0}^{*}, x_{0}^{* *}\right)\right\} .
$$

Then the problem (18) (19) has at least $4 k$ solutions.

Remark 3.1. The half-period $T\left(x_{0}\right)$ is given by the formula

$$
T\left(x_{0}\right)=\frac{1}{\sqrt{2}} \int_{x_{0}}^{x_{1}} \frac{\mathrm{d} s}{\sqrt{G\left(x_{0}\right)-G(s)}},
$$

where $x_{1}$ is the first larger than $x$ zero of the $s$-function $G\left(x_{0}\right)-G(s)$.

EXAmple 1. Consider equation (18), where $g(x)=-x\left(x-p_{1}\right)\left(x-p_{2}\right)\left(x-p_{3}\right) \times$ $\left(x-p_{4}\right), p_{1}=-4, p_{2}=-1.5, p_{3}=2, p_{4}=5$ together with conditions (19), where $a=0, b=1$.

$$
I_{1}=\left[x_{0}^{*} ; x_{0}^{* *}\right]=[-4 ;-2.21933], \quad I_{2}=\left[x_{1}^{*} ; x_{1}^{* *}\right]=[2.88632 ; 3.82839] .
$$


PERIOD ANNULI AND MULTIPLE SOLUTIONS FOR TWO-POINT BVP

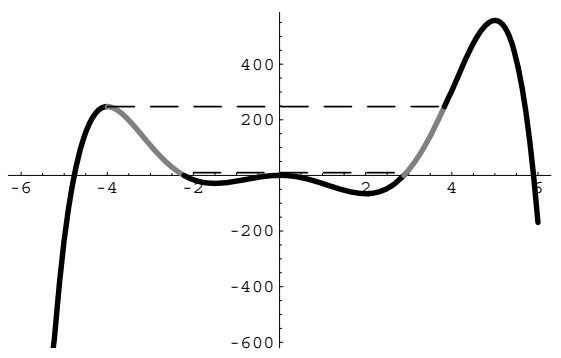

FIGURE 3.

Primitive $G(x)$.

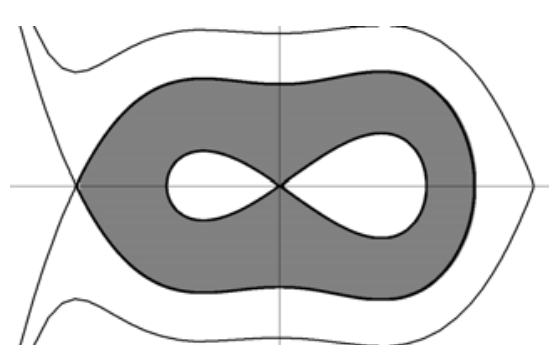

Figure 4.

The respective phase portrait.

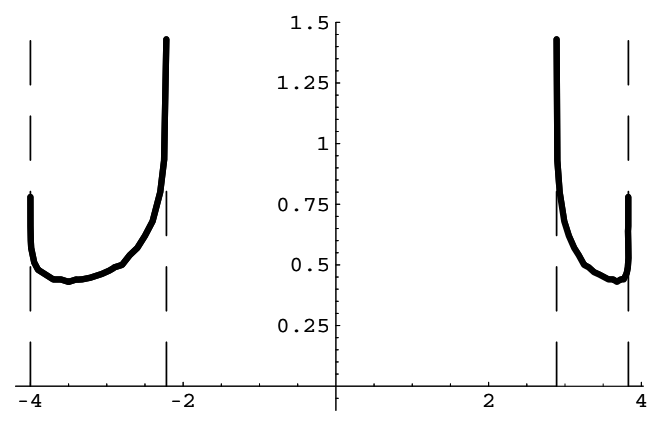

FIGURE 5 .

Time $T\left(x_{0}\right)$, where $x_{0}$ is of $I_{1}$ and of $I_{2}$, respectively.

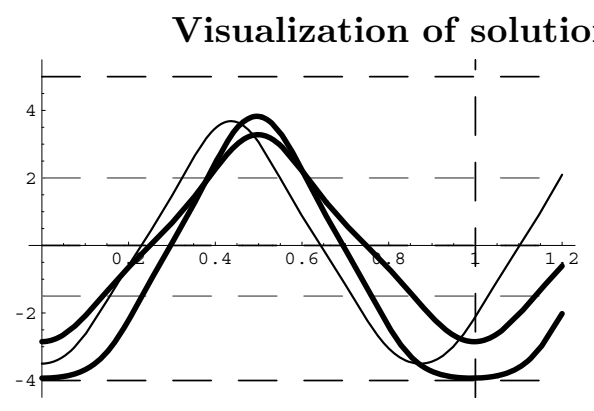

Figure 6

Solutions: $x(0)=-3.93, x(0)=-3.5$, $x(0)=-2.85$. Thin line-solution with a minimal period.

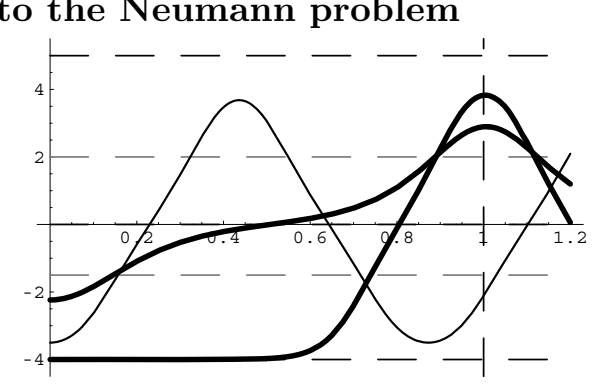

FIGURE 7.

Solutions: $x(0)=-3.9999995, x(0)=-3.5$, $x(0)=-2.237$. Thin line - solution with a minimal period. 


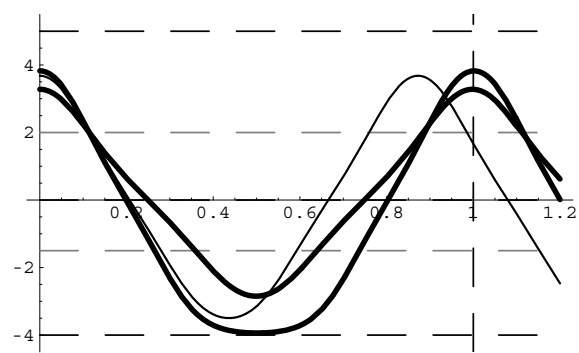

FiguRe 8.

Solutions: $x(0)=3.28, x(0)=3.68, x(0)=$ 3.8255. Thin line - solution with a minimal period.

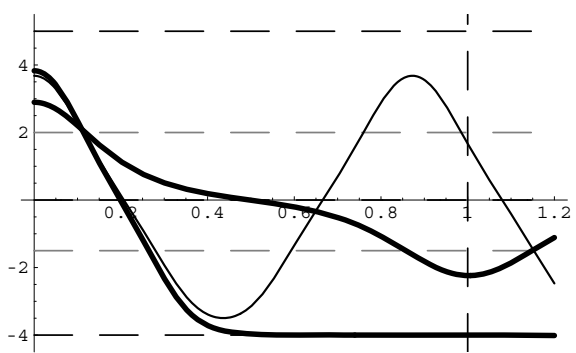

Figure 9.

Solutions: $x(0)=2.896, x(0)=3.68$, $x(0)=3.8283910155$. Thin line-solution with a minimal period.

Theorem 3.2. Suppose equation (18) has multiple period annuli $C_{i}, i=1,2, \ldots$ $\ldots, m$. Let $C_{i x}$ be intersection of a period annulus $C_{i}$ with the $x$-axis (this intersection is a sum of two open intervals $I_{i 1}$ and $\left.I_{i 2}\right)$. Let the meanings of $T_{i}\left(x_{0}\right)$ and $T_{i \min }$ be the same. Let also

$$
k_{i} T_{i_{\min }}<b-a<\left(k_{i}+1\right) T_{i_{\min }} .
$$

Then the problem (18), (19) has at least $4\left(k_{1}+\ldots+k_{m}\right)$ solutions.

ExAmple 2. Consider equation (18), where $g(x)=-10 x\left(x-p_{1}\right)\left(x-p_{2}\right) \times$ $\left(x-p_{3}\right)\left(x-p_{4}\right)\left(x-p_{5}\right)\left(x-p_{6}\right)\left(x-p_{7}\right)\left(x-p_{8}\right), p_{1}=-3, p_{2}=-2.2, p_{3}=-1.9$, $p_{4}=-0.8, p_{5}=0.3, p_{6}=1.5, p_{7}=2.3, p_{8}=2.9$ together with conditions (19), where $a=0, b=1$.

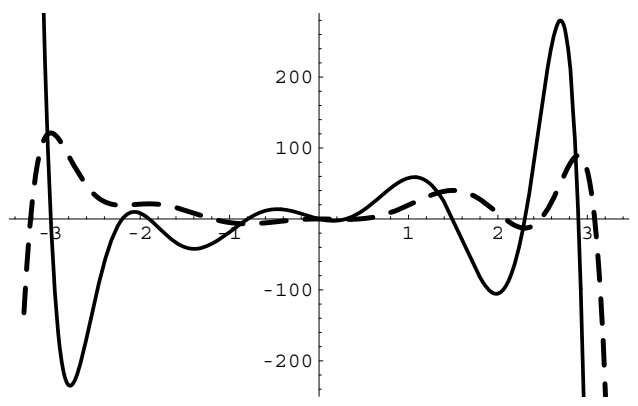

FiguRE 10 .

Primitive function $G(x)$.

$$
\begin{array}{rlrl}
I_{1} & =\left[x_{0}^{*} ; x_{0}^{* *}\right]=[-2.55 ;-2.33356], & I_{2} & =\left[x_{1}^{* *} ; x_{1}^{*}\right]=[1.03516 ; 1.5], \\
I_{1}=\left[x_{0}^{*} ; x_{0}^{* *}\right]=[-1.9 ;-1.1904], & I_{2}=\left[x_{1}^{* *} ; x_{1}^{*}\right]=[0.443116 ; 1.03516] .
\end{array}
$$


PERIOD ANNULI AND MULTIPLE SOLUTIONS FOR TWO-POINT BVP

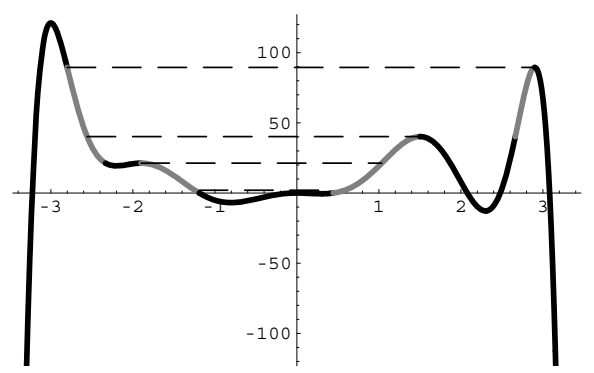

Figure 11.

Primitive function $G(x)$.

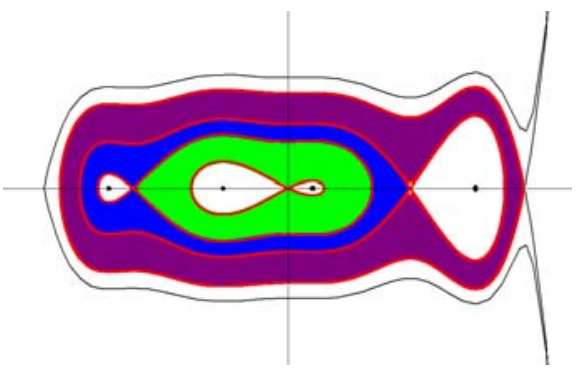

Figure 12.

The phase plane.

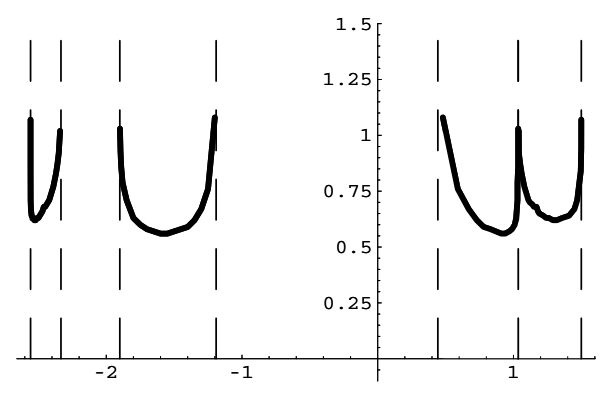

Figure 13.

$T\left(x_{0}\right)$ dependence for outer and inner period annuli.

\section{Visualization of solutions to the Neumann problem (for period annuli)}

Remark 3.2. Thick line everywhere represents a solution with a minimal period in a period annulus.

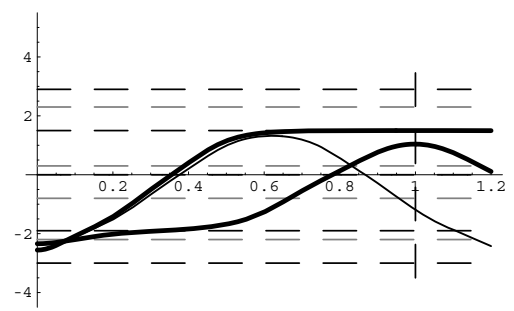

a.

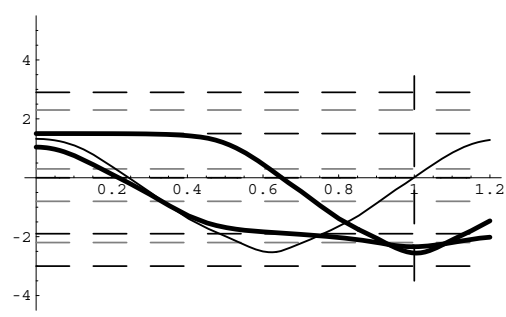

b.

FIGURE 14.

a. Solutions: $x(0)=-2.55760909, x(0)=-2.53, x(0)=-2.3416$.

b. Solutions: $x(0)=1.49978, x(0)=1.32, x(0)=1.04$. 


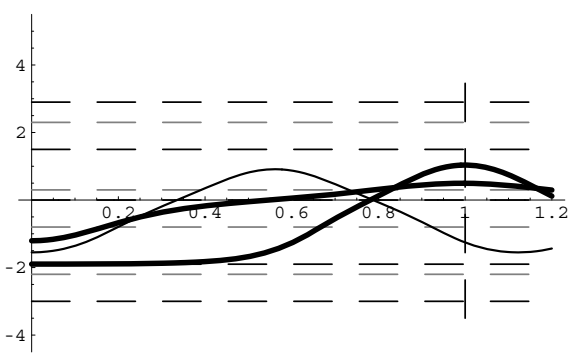

a.

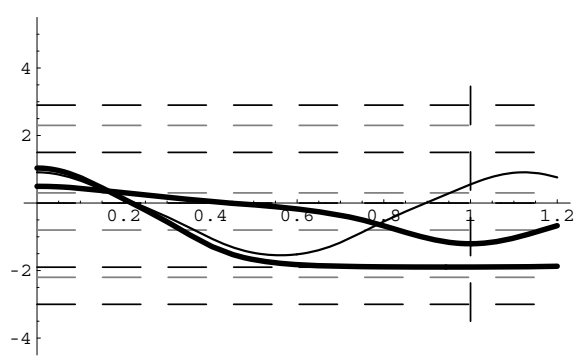

b.

FiguRE 15.

a. Solutions: $x(0)=-1.8975, x(0)=-1.55, x(0)=-1.206$.

b. Solutions: $x(0)=0.495, x(0)=0.91, x(0)=1.03514$.

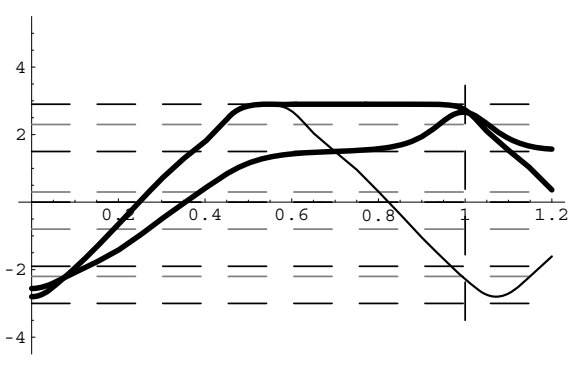

a.

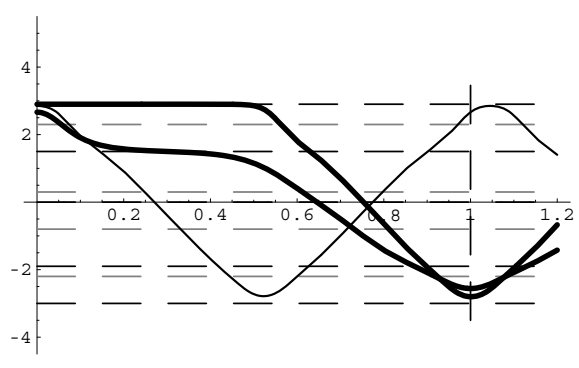

b.

Figure 16.

a. Solutions: $x(0)=-2.801266808983321, x(0)=-2.8, x(0)=-2.5585$.

b. Solutions: $x(0)=2.66385, x(0)=2.85, x(0)=2.8999999999999$.

\section{Equation $X^{\prime \prime}+F(X) X^{\prime 2}+G(X)=0$. Reduction to} a conservative equation

It was shown by $\mathrm{S}$ a b a t in i 4 that equation (7) can be reduced to the form $u^{\prime \prime}+h(u)=0$ by the following transformation. Let

$$
F(x)=\int_{0}^{x} f(s) \mathrm{d} s \text { and } G(x)=\int_{0}^{x} g(s) \mathrm{d} s .
$$

Introduce

$$
u:=\Phi(x)=\int_{0}^{x} e^{F(s)} \mathrm{d} s .
$$


Since $\frac{\mathrm{d} u}{\mathrm{~d} x}>0$, this is one-to-one transformation and the inverse $x=x(u)$ is well defined.

Lemma 4.1 (44, Lemma 1). The function $x(t)$ is a solution to (7) if and only if $u(t)=\Phi(x(t))$ is a solution to

$$
u^{\prime \prime}+g(x(u)) e^{F(x(u))}=0 .
$$

Denote

$$
H(u)=\int_{0}^{u} g(x(s)) e^{F(x(s))} \mathrm{d} s .
$$

The existence of periodic solutions depends entirely on properties of the primitive $H$.

Let us state some easy assertions ([1], [2]) about equation (7), the equivalent system

$$
x^{\prime}=y, \quad y^{\prime}=-f(x) y^{2}-g(x)
$$

and the system

$$
x^{\prime}=y, \quad y^{\prime}=-g(x) .
$$

Proposition 4.1. Critical points and their character are the same for systems (25) and (24).

Consider a system

$$
u^{\prime}=v, \quad v^{\prime}=-g(x(u)) e^{F(x(u))},
$$

which is equivalent to equation (23).

Proposition 4.2. Critical points $(x, 0)$ and $(u(x), 0)$ of systems (25) and (26), respectively, are in 1-to-1 correspondence and their characters are the same.

Proposition 4.3. Periodic solutions $x(t)$ of equation (7) turn to periodic solutions $u(t)=\Phi(x(t))$ of equation (23) by transformation (22).

Proposition 4.4. Homoclinic solutions of equation (7) turn to homoclinic solutions of equation (23) by transformation (22).

Proposition 4.5. Let $p_{i}$ be a zero of $g(x)$. The equality

$$
g_{x}\left(p_{i}\right)=\left.g_{u}(x(u)) e^{F(x(u))}\right|_{u=p_{i}}
$$

is valid.

P r o of. By calculation the derivative. 


\section{The Liénard type equations}

Consider equation (7), where $g(x)=-x\left(x^{2}-p^{2}\right)\left(x^{2}-q^{2}\right), f(x)=a x, p=0.25$, $q=0.4, a=8$. Maxima of the function $G$ are at the points $-q, 0$ and $q$. Since

$$
G(p)=G(-p)<G(0),
$$

equation $x^{\prime \prime}+g(x)=0$ has not nontrivial period annuli. This is shown in Fig. 18]a.

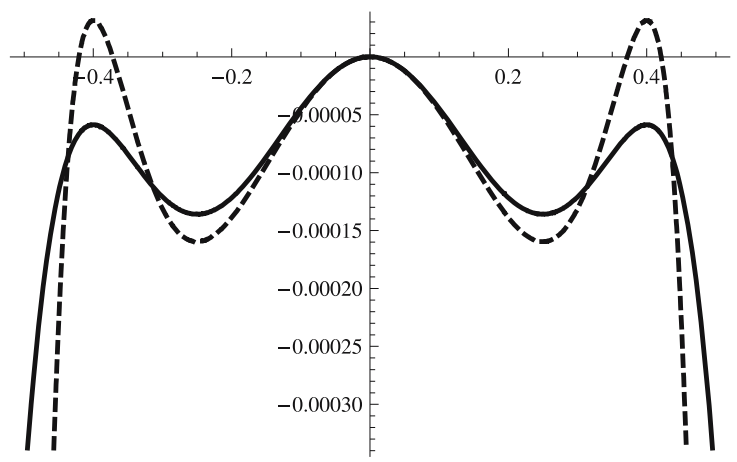

Figure 17.

$G(x)$-solid, $H(u)$-dashed (rescaled).

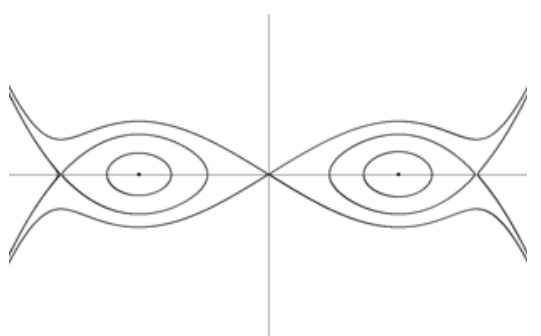

a.

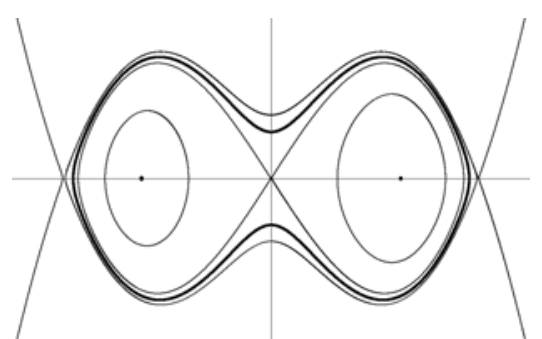

b.

Figure 18 .

a. Phase plane for $x^{\prime \prime}+g(x)=0$.

b. Phase plane for equation (7).

The function $H(u)$ has maxima at $u(-q), 0$ and $u(q)$. Since

$$
H(u(q))=H(u(-q))>H(0)=0,
$$

equation (7) has a nontrivial period annulus as seen in Fig. 18b. 


\section{The Neumann problem for the Liénard type equations}

The analogues of Theorems 3.1 and 3.2 are valid if instead of the function $G(x)$ the function

$$
H(u)=\int_{0}^{x(u)} g(\xi) e^{2 F(\xi)} \mathrm{d} \xi
$$

is considered, where $x(u)$ is the inverse to $u(x)$ defined by (22).

\section{REFERENCES}

[1] ATSLEGA, S.: On solutions of the Liénard type equations, in: Proc. Mathematics. Differential Equations, Vol. 7, Univ. of Latvia, Institute of Math. and Comp. Sci., 2007, pp. 53-60, http://www. lumii.lv/Pages/LUMII-2007/Sbornik-2007-english.htm.

[2] ATSleGA, S.-SADYRBAEV, F.: Period annuli in the Liénard type equation, Int. J. Pure Appl. Math. 44 (2008), 117-123.

[3] SABATINI, M.: Liénard limit cycles enclosing period annuli, or enclosed by period annuli, Rocky Mountain J. Math. 35 (2005), 253-266.

[4] SABATINI, M.: On the period function of $x^{\prime \prime}+f(x) x^{\prime 2}+g(x)=0$, J. Differential Equations 196 (2004), 151-168.

Received September 14, 2008

Daugavpils University

Department of Mathematics and

Natural Sciences

Parades iela 1

LV-5400 Daugavpils

LATVIA

E-mail: felix@latnet.lv svetlana.og@inbox.lv 